\title{
International Workshop on Thin Films Held in Iran
}

The International Workshop on the Physics and Technology of Thin Films was hosted by the Physics Department of Sharif University of Technology in Tehran, Iran, February 22-March 6, 2003. The workshop was organized by A.Z. Moshfegh of Sharif University in collaboration with an international advisory committee consisting of S.C. Kashyap (Indian Institute of Technology, New Delhi), M. Ohring (Stevens Institute of Technology, Hoboken, N.J.), G. Ottaviani (Università degli Studi di Modena, Italy), and A. Zvezdin (General Physics Institute, Russian Academy of Science). Participants attended from 23 countries. The formal workshop program consisted of

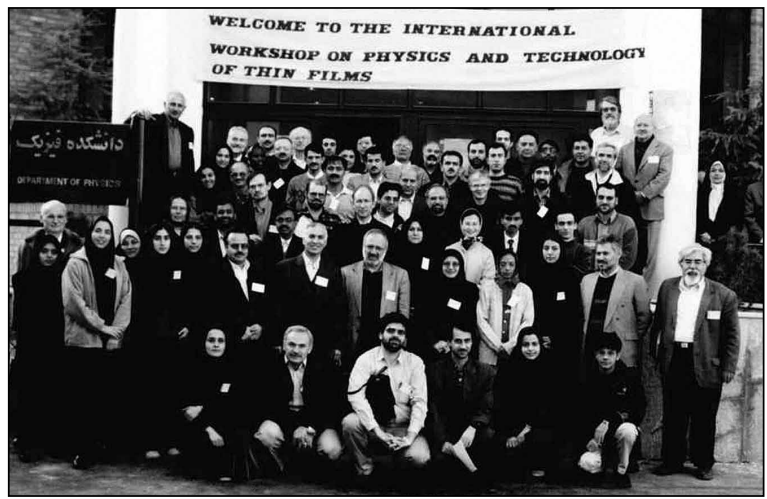

Conference participants in front of the Physics Department building of Sharif University. $\sim 30$ invited talks as well as a similar number of contributed seminars, plus a few poster presentations. Many of the invited talks and presentations were tutorial in nature, reviewing topics of continuing interest (see sidebar). Between lectures, graduate students of physics and materials science at Sharif University interacted with the visiting scientists, and meeting attendees visited various thin-film and surface-analysis research laboratories. Tours rounded out the technical portion of the program, and included visits to the TABA Cutting Tools Manufacturing Company in Karaj where drills and carbide inserts are coated with TiN by physical vapor deposition and chemical vapor deposition methods, respectively, and to Isfahan Optical Industries in Isfahan where lenses are coated with optical coatings.

Sharif University of Technology is one of the leading technological universities in the Middle East and central Asia. Nevertheless, the overall level of research lags that of the West, and research facilities are modest in most cases. Attempts to redress this situation are being made, however. For example, the Physics Department has recently acquired a modern

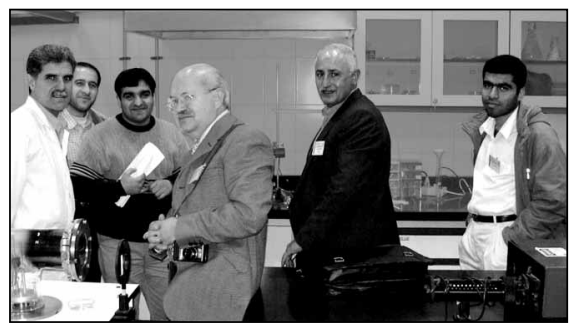

Conference attendees during one of the tours of thin-film laboratories.

Auger/x-ray photoelectron spectroscopy/ ultraviolet photoelectron spectroscopy (hemispherical electron analyzer) spectrometer with the capability of in situ analysis of films deposited under ultrahigh-vacuum conditions. Women compose almost half of the student body at the school. Virtually all of the science and engineering faculty members were educated in the West, with about $80 \%$ receiving doctorate degrees from U.S. graduate schools. There are a number of collaborations between institutions in the United States and Europe and professors at Sharif University.

The Second International Workshop on the Physics and Technology of Thin Films has been tentatively scheduled to take place in Prague, Czech Republic, in 2006.

MILT OHRING

International Scientific Organizing Committee

\section{International Workshop on the Physics and Technology of Thin Films: Partial List of Presentations}

M. Farle (Universität Duisburg, Germany): Magnetism in Thin Films; Exchange Coupling and Curie Temperature in Multilayers

M. Henini (University of Nottingham, U.K.): Structural and Optical Properties of Self-Assembled Quantum Dots Grown by Molecular-Beam Epitaxy

H.v. Kaenel (Politecnico di Milano, Italy): Epitaxy; Superlattices

S.C. Kashyap (Indian Institute of Technology-New Delhi): Modified Amorphous Thin Films; GMR in Thin Films

S.H. Keshmiri (Ferdowsi University, Iran): Diffusion of Atomic Hydrogen and Passivation of Structural Defects in Silicon and Transparent Conducting Thin Films

S.H. Keshmiri and M. Mirsalehi (Ferdowsi University, Iran): Multilayer Thin-Film Optical Filters: Design, Fabrication, and Applications

S.K. Kulkarni (University of Pune, India): XPS Analysis of Thin Films; Thin-Film Analysis Using Synchrotron Radiation

J.G. Lin (National Taiwan University): Physics and Applications of $\mathrm{YBa}_{2} \mathrm{Cu}_{3} \mathrm{O}_{7} / \mathrm{La}_{0.7} \mathrm{Sr}_{0.3} \mathrm{MnO}_{3}$ Heterostructures
D. Acosta (Universidad Nacional Autónoma, Mexico): Photocatalysis in $\mathrm{TiO}_{2}$ Thin Films

A.Z. Moshfegh (Sharif University of Technology, Iran): Vacuum Technology: Principles and Applications; PVD Growth Methods: Physics and Technology

M. Ohring (Stevens Institute of Technology, USA): Structure of Polycrystalline Thin Films; Failure and Reliability of Thin Films in Electronic Devices

A.I. Popov (Moscow University of Electronics-MIET, Russia): Magneto-Optics of Thin Films and Nanostructures; Microscopic Mechanisms of Magneto-Optical Activity in Epitaxial Garnet Films

P. Soukiassian (Centre Energie Atomique and Université Paris-Sud, France): Nanostructure and Nanochemistry on Silicon Carbide Surfaces; Atomic Structure and Electronic Properties of Advanced Semiconductor Surfaces

M. Wuttig (Rheinisch-Westfälische Technische HochschuleAachen, Germany): The Quest for New Phase-Change Media in Ultrafast Optical Data Storage; In Situ Studies of Gasochromic Switching 